\title{
The Utility of Molecular Imaging for Investigating Patients with Visual Hallucinations
}

W e read with interest the recent article entitled "Distinguishing Neuroimaging Features in Patients Presenting with Visual Hallucinations" by Winton-Brown et al. ${ }^{1}$ They described the features of dementia with Lewy bodies (DLB) on multiplanar T1-weighted MR imaging. ${ }^{1}$ In our practice, we find the diagnosis of DLB challenging with anatomic imaging alone. Molecular imaging can be helpful for differentiating DLB from other causes of visual hallucinations and dementia. ${ }^{2-4}$ We present examples of the imaging findings of DLB by using nigrostriatal dopamine terminal imaging with dopamine-transporter single-photon emission CT (DaTscan) with the radioligand $\left[{ }^{123} \mathrm{I}\right] \mathrm{FP}-\mathrm{CIT}$ (GE Healthcare, Buckinghamshire, United Kingdom) and FDG-PET. These imaging techniques may be considered in addition to anatomic imaging in cases in which a diagnosis of DLB is suspected but remains unclear.

DaTscan is used for the early detection of Parkinson disease, to differentiate essential tremor and psychogenic-/neuroleptic-induced parkinsonism from presynaptic parkinsonian syndromes and to distinguish DLB from other forms of dementia. Nigrostriatal dopaminergic denervation is a crucial feature in Parkinson disease, parkinsonian disorders and DLB. $\left[{ }^{123} \mathrm{I}\right]$ FP-CIT has a high affinity for the dopamine transporter (DaT) and is used to visualize dopaminergic nerve terminals. DaTscan involves the acquisition of SPECT images 3-6 hours after a single injection of $\left[{ }^{123} \mathrm{I}\right] \mathrm{FP}-\mathrm{CIT}$ at a recommended dose of 111-185 MBq by using a dual-headed (or greater) gamma camera. In a normal DaTscan, the striatum is visible as symmetric, comma-shaped regions. Both the caudate and putamen show high uptake compared with the background (Fig 1A). In $\mathrm{DLB}$, there is typically bilateral loss of DaT binding, predominantly in the putamen and caudate, more symmetric than in Parkinson disease (Fig $1 B$ ). A Phase III study by McKeith et $\mathrm{al}^{2}$ showed that DaTscan has a mean sensitivity of $77.7 \%$ for detecting clinically probable DLB and a specificity of $90.4 \%$ for excluding other causes of dementia, principally Alzheimer disease $(\mathrm{AD})$.

FDG-PET is a useful imaging technique in differentiating

http://dx.doi.org/10.3174/ajnr.A4937

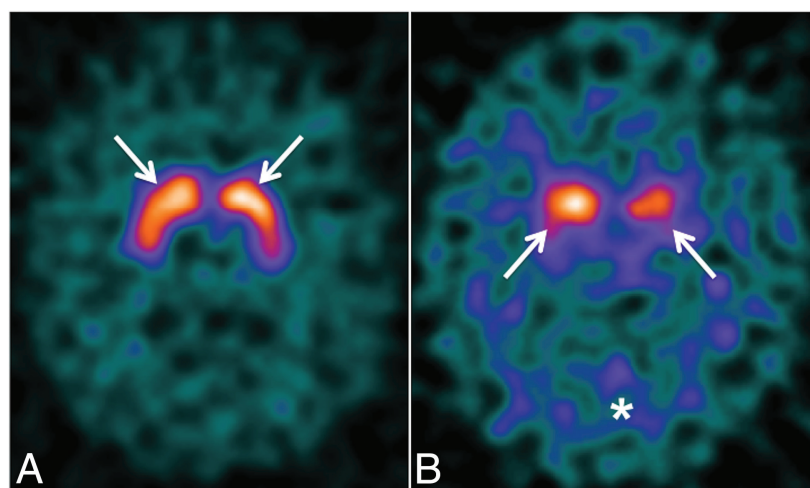

FIG 1. A, Normal DaTscan findings with normal background activity. The arrows show normal radiotracer uptake within the caudate nuclei and putamen bilaterally. B, Abnormal DaTscan findings in a 68-yearold woman with visual hallucinations and cognitive impairment. There is reduced uptake within the caudate nuclei and putamina (arrows) bilaterally with increased background activity (asterisk). In the clinical context, these findings are consistent with DLB.

primary neurodegenerative disorders. The FDG metabolic signature of DLB is bilateral parietal and posterior temporal hypometabolism, posterior cingulate gyral hypometabolism, and involvement of the occipital lobes, which are spared in $\mathrm{AD}$ (Fig 2). ${ }^{3}$ Medial occipital lobe hypometabolism is a key feature of DLB that discriminates it from $\mathrm{AD},{ }^{4}$ with a reported sensitivity and specificity of $92 \%$. If the occipital cortex is not involved, $\mathrm{DLB}$ and $\mathrm{AD}$ cannot be distinguished on the basis of their FDG metabolic pattern. ${ }^{3}$ When clinical and FDG-PET findings are indeterminate, DaTscan is complementary.

In conclusion, the integration of molecular imaging, including DaTscan and FDG-PET, can be useful in establishing a diagnosis of dementia with Lewy bodies in patients presenting with visual hallucinations.

Disclosures: Ronan P. Killeen-UNRELATED: Consultancy: Novartis, Comments: consultancy related to review of software for atrophy measures in multiple sclerosis; single fee under \$1000; Royalties: Springer, Comments: FDG PET/CT in Clinical Oncology (book) by Jasna Mihailovic, Ronan P. Killeen, and Stanley J. Goldsmith, 2012; Stock/Stock Options: Dublin CyberKnife Financing Limited, Comments: owns shares in this company; it runs a CyberKnife for treatment of primarily brain tumors in Hermitage Medical Clinic, Dublin, Ireland. 


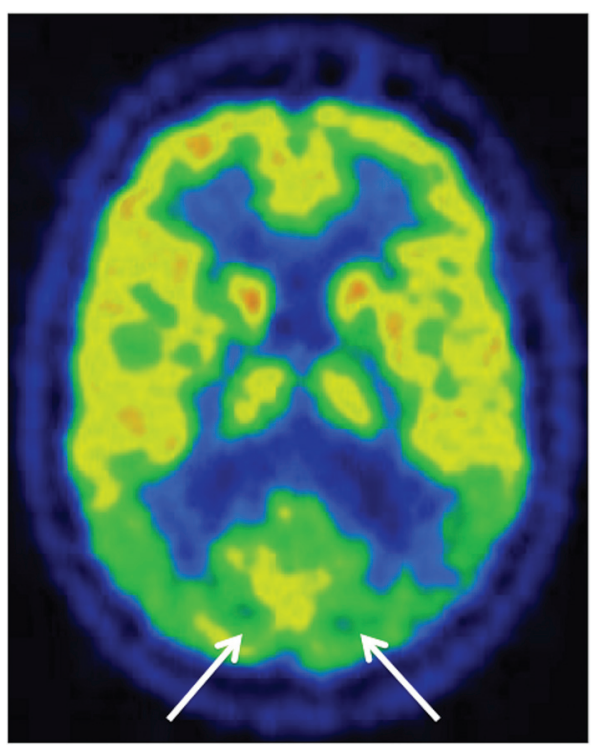

FIG 2. FDG-PET in the same patient as Fig $1 B$ shows marked bilateral hypometabolism in the medial occipital lobes, which is a typical finding in DLB. ${ }^{3}$ These features distinguish DLB from AD; AD typically shows hypometabolism in the temporal-parietal regions and posterior cingulate gyrus but with normal medial occipital metabolism.

\section{REFERENCES}

1. Winton-Brown TT, Ting A, Mocellin R, et al. Distinguishing neuroimaging features in patients presenting with visual hallucinations. AJNR Am J Neuroradiol 2016;37:774-81 CrossRef Medline

2. McKeith I, O'Brien J, Walker Z, et al; DLB Study Group. Sensitivity and specificity of dopamine transporter imaging with ${ }^{123}$ I-FP-CIT SPECT in dementia with Lewy bodies: a phase III, multicentre study. Lancet Neurol 2007;6:305-13 CrossRef Medline

3. Brown KJ, Bohnen NI, Wong KK, et al. Brain PET in suspected dementia: patterns of altered FDG metabolism. Radiographics 2014; 34:684-701 CrossRef Medline

4. Ishii K. PET approaches for diagnosis of dementia. AJNR Am J Neuroradiol 2014;35:2030-38 CrossRef Medline

(1)S. Clifford

(D) Y.M. Purcell

Department of Radiology St. Vincent's University Hospital

Dublin, Ireland (D) R.P. Killeen

Department of Radiology St. Vincent's University Hospital Dublin, Ireland Department of Radiology Royal Victoria Eye and Ear Hospital Dublin, Ireland 